\title{
Pratiques
}

Linguistique, littérature, didactique

\section{À la croisée des pans de cohésion textuelle : les variations lexicales du Règne de l'esprit malin, de C. F. Ramuz}

\section{Vincent Verselle}

\section{OpenEdition}

Journals

Édition électronique

URL : http://journals.openedition.org/pratiques/3469

DOI : $10.4000 /$ pratiques.3469

ISSN : 2425-2042

Éditeur

Centre de recherche sur les médiations (CREM)

\section{Édition imprimée}

Date de publication : 15 décembre 2012

Pagination : 83-96

Référence électronique

Vincent Verselle, «À la croisée des pans de cohésion textuelle : les variations lexicales du Règne de l'esprit malin, de C. F. Ramuz », Pratiques [En ligne], 155-156 | 2012, mis en ligne le 18 décembre 2017, consulté le 20 avril 2019. URL : http://journals.openedition.org/pratiques/3469 ; DOI : 10.4000/ pratiques.3469 


\title{
À la croisée des pans de cohésion textuelle : les variations lexicales du Règne de l'esprit malin, de C. F. Ramuz
}

\author{
Vincent Verselle
}

Université de Lausanne

\author{
Je continue par-dessus ce $1{ }^{\text {er }}$ mars ce travail difficile et dur, dont je ne sais \\ encore ce qu'il résultera [;] \\ travail aussi dans des gravières d'écritures \\ dans des éboulements de lignes \\ dans des déplacements de masses par le jeu \\ des lumières et des ombres du sentiment [;] \\ morne travail \\ recherche [;] \\ trouver le mot[,] sentir vivre et battre le mot \\ comme l'artère sous le doigt[.] ${ }^{(1)}$
}

Quand on lit pareille confidence d'écrivain, il semble bien qu'on doive se représenter le processus d'élaboration d'un texte comme étant avant tout une quête inquiète de mots, voire $d u$ mot, le mot « juste». Le singulier employé ici par Ramuz, comme celui qui est de mise dans des expressions courantes (« Je ne trouve pas le bon mot»), pourrait cependant donner une image " atomisée » de la production de discours, où le mot « juste » serait, à la limite, celui qui se suffit à lui-même pour jouer son rôle (expressif, référentiel, persuasif, etc.). La réalité est, bien entendu, tout autre :

[...] le sujet parlant ne communique généralement pas plus par mots isolés que par phrases ou par propositions non liées. Toute communication humaine se fait au moyen d'énoncés suivis, oraux ou écrits, qui vont d'un simple mot oral à plusieurs volumes écrits. (Adam 1999: 24)

Autrement dit, cette communication se concrétise nécessairement sous la forme d'un texte. Dans cette perspective, la pesée des mots lors d'une production de discours relève alors fondamentalement des normes textuelles qui président à ce proces-

(1) Entrée datée du $1{ }^{\mathrm{er}}$ mars 1921 du journal de Ramuz (2005: 7); 1'auteur élabore à ce moment un roman (resté inédit de son vivant) intitulé " Travail dans les gravières ", d'où certaines métaphores qu'il emploie ici. 
sus même. En ce sens, l'image des « gravières d'écritures » est on ne peut plus parlante : le mot «juste» est en définitive celui qui empêche (avec d'autres) le texte de s'écrouler, dans la mesure où il participe à l'organisation de cette totalité cohésive que l'on reconnaît comme texte. Mais plus encore, suivant cette interaction entre le mot et le texte, le singulier de mise tend à disparaître en faveur du pluriel, car si la totalité visée détermine le choix des parties en cours de route, chaque partie affecte pareillement la structuration du tout. Il en résulte ainsi que le mot juste cache une pluralité de mots potentiellement justes, et que ces options divergentes, si elles étaient réalisées, aboutiraient non pas à une désorganisation, mais à une réorganisation fine de la cohésion textuelle.

En ouvrant un passage vers la fabrique d'un texte, l'approche génétique apporte son propre éclairage sur les liens entre la variation lexicale et les opérations qui sous-tendent le processus de (ré)écriture, et en particulier les opérations de liage visant à élaborer un ensemble cohésif. L'étude des manuscrits révèle ainsi les divers " essayages » de mots dans un segment particulier et permet d'évaluer les implications textuelles de tels phénomènes de variation; mais ceux-ci se prolongent bien au-delà de l'étape du manuscrit, puisqu'ils peuvent survenir lors de la phase éditoriale de la genèse d'une œuvre, lorsque l'écrivain (entre autres acteurs de cette genèse) décide, à l'occasion d'une réédition, de modifier son texte. Or il se trouve que C. F. Ramuz, lui, est tout à fait coutumier de ce type de remaniements, notamment en ce qui concerne ses proses longues : ainsi par exemple du Règne de l'esprit malin, « histoire » parue originellement en revue en 1914, et qui a connu cinq rééditions entre 1917 et 1946, chacune comportant son lot (parfois très important) de modifications.

Dans les pages suivantes, je me propose alors d'observer quelques changements entre versions publiées qui portent sur le matériau lexical, et plus particulièrement des faits de substitution d'un mot par un autre, afin de montrer dans quelle mesure ces variations, malgré leur caractère extrêmement localisé, ne trouvent pas leur motivation à un niveau strictement « local » (réagencement syntagmatique ou propositionnel), mais qu'elles procèdent bien d'une élaboration textuelle du sens (ce qui a souvent pour corollaire que, dans un segment donné, une telle substitution intervient parallèlement à d'autres modifications, dont il importe de tenir compte). Plus encore, l'étude de ces variations apportera un éclairage sur des caractéristiques essentielles de la textualité : on constatera en effet que les unités lexicales se trouvent souvent à la confluence de plusieurs forces cohésives - à la manière de l'espace central d'un diagramme de Venn -, forces qui agissent sur des portées différentes, et que la variation vient pondérer à sa manière. On conçoit aisément que ces modifications lexicales mettent principalement en jeu la dimension sémantique de la cohésion textuelle (mais pas uniquement, comme on le verra), que l'on va alors saisir dans toute sa stratification ${ }^{(2)}$.

\section{Le mot frontière : tensions entre le local et l'étendu}

Les liages sémantiques se déploient notamment sous la forme d'isotopies, souvent plurielles au sein d'un segment textuel, œuvrant à l'échelle de la proposition

(2) Ce corpus ciblé provient de l'édition critique des œuvres complètes de Ramuz (volume 23, Genève, Slatkine, 2012), projet piloté par Roger Francillon et Daniel Maggetti, directeur du Centre de recherches sur les lettres romandes de l'Université de Lausanne. Outre le texte de l'édition originale du Règne de l'esprit malin (1917) publié sur le support papier du volume, ce dernier présente sur CD-ROM les comparaisons entre les six éditions, par paires successives - dispositif de lecture qui a été réalisé à partir du logiciel d'alignement textuel MEDITE, adapté par Rudolf Mahrer aux besoins de cette édition. 
et/ou à celle d'une unité textuelle de rang supérieur, selon un partage de zones que la variation lexicale peut redéfinir.

A propos de la notion d'isotopie, il faut rappeler que celle-ci doit se comprendre comme un parcours de sens, qui est le produit tout à la fois de certaines composantes de la structure du texte et d'une activité interprétative, puisque « ce n'est pas seulement la récurrence de sèmes donnés qui constitue l'isotopie, mais la présomption d'isotopie qui permet d'actualiser des sèmes, voire les sèmes » (Rastier 1987 : 82 ; l'auteur souligne). Or si l'on pose que le premier interprète d'un texte est son producteur même ( $c f$. Grésillon $1988: 112$ ), on peut faire l'hypothèse que le mouvement de l'écriture est soumis pareillement à cette "présomption » d'un parcours à accomplir, le long d'un paradigme lexical susceptible de produire des récurrences sémiques en suffisance. C'est ainsi que, selon Doquet-Lacoste, l'observation des activités scripturales en contexte scolaire fait apparaître que «c'est bien le versant lexical de la langue, et le déploiement du champ sémantique des mots, qui sert d'appui à l'expansion textuelle " (Doquet-Lacoste 2008 : 131). Mais pareil constat vaut évidemment pour les locuteurs qui ont parfait leur apprentissage, tel un auteur soucieux de " faire image », comme Ramuz, et qui pour ce faire décide d'exploiter des décalages sémantiques propices à la création d'une métaphore filée. L'intérêt ici d'une écriture qui vise le figural réside dans le fait que, nécessairement, celle-ci doit articuler (au moins) deux isotopies, ce qui n'est pas sans effet sur le jeu des forces cohésives à l'œuvre dans un segment de ce type : la perspective génétique montre en effet que les unités lexicales, durant ce processus, sont des lieux que se disputent des champs d'attraction sémantique pluriels, l'écrivain arbitrant à chaque choix en faveur de l'un ou de l'autre. Voici le premier paragraphe de la description d'un paysage brumeux, dans la première version éditée du Règne de l'esprit malin :

A cause du brouillard, on ne voyait plus le village. Cet étage d'en bas, où il était bâti, était recouvert comme d'un linge, sous lequel tout disparaissait, à part la pointe du clocher, qui en sortait par une déchirure. Et plus loin, à l'endroit où s'ouvrait la vallée, le même voile s'étendait, et, partant de ce versant-ci, allait s'attacher au versant d'en face, dont il ne laissait voir que la partie d'en haut. Ainsi le vide était comblé. Mais on le devinait quand même. Et qu'il y avait l'air dessous, cela se devinait aussi, à cause d'un mouvement qui passait quelquefois, glissant à sa surface comme une vague sur un lac ; puis un lambeau en était arraché, qui venait lentement vers vous. (Ramuz 1914, 1 : 557-558)

Clairement (si l'on peut dire), le déclencheur du déploiement descriptif est le sème inhérent ${ }^{(3)} /$ visibilité/ qui participe à former l'étiquette sémantique du nom brouillard, et que l'on retrouve immédiatement dans le verbe voir, bien entendu, puis dans le verbe recouvrir, ainsi que dans les autres mots soulignés ici en gras, avec des va-et-vient entre /visibilité (-)/ et /visibilité $(+) /{ }^{(4)}$. Quant à l' « image », elle va prendre forme par le biais du décalage sémantique que produit la récurrence du sème/tex-

(3) La distinction entre sèmes inhérents et afférents a été introduite par Rastier. Les premiers relèvent du système fonctionnel de la langue - mais c'est là, selon Rastier, la « langue restreinte des grammaires » (1987:63); le système linguistique « au sens large [...] comprend, outre le système fonctionnel de la langue, des normes socialisées à l'œuvre dans les textes », dont dépendent les sèmes afférents, « socialement normés » (ibidem).

(4) Il est difficile de rattacher un sème /visibilité/ au nom surface; mais le cotexte où celui-ci apparaît, et notamment la relation anaphorique tissée entre le syntagme «sa surface » et l'antécédent "le même voile », a pour résultat de rendre saillant le trait inhérent /limitation/ dans le signifié du mot, trait qui paraît être alors une modulation du sème /visibilité/ (entrave au regard). 
tile/ (mots en italique ${ }^{(5)}$ ), décalage que le texte ici prend le soin d'annoncer (par le marqueur de rupture d'isotopie comme) au moment d'introduire le nom linge. On constate que c'est d'abord la syntaxe qui a la charge d'opérer l'association entre les deux réseaux sémantiquement disjoints, par quoi linge se voit doté de rôles sémantiques (instrumental et locatif) dans le cadre des procès dénotés par recouvrir et disparaître (de même pour le rôle de locatif que la syntaxe fait endosser à déchirure par rapport à sortir). Mais le lexique prend ensuite le relais, et de façon particulièrement efficace, à travers la reprise anaphorique de linge par le nom voile : dans la structure sémantique du paragraphe en effet, ce mot est un point d'articulation essentiel, puisque son signifié fait converger les deux réseaux, par le cumul des sèmes (inhérents) /textile/ et/visibilité (-)/. Dès lors, dans la phrase où ce nom fonctionne comme sujet de deux verbes, la cohésion joue tant sur l'articulation syntaxique (sujet-prédicat) que sur les récurrences sémiques avec les verbes s'étendre et s'attacher, d'une part, et, d'autre part, avec (laisser) voir. (Il faut signaler toutefois que, si cette " ouverture " sémantique confère au nom voile un haut pouvoir cohésif dans ce contexte, celle-ci va, de façon paradoxale, être la cause probable du remplacement de ce mot lors d'une phase de réécriture, comme on va le voir plus loin.)

D'autres isotopies, plus locales, structurent ce segment de texte (comme l'isotopie /construction/ formée par village, bâti, clocher). Il en est une qui mérite un peu plus d'attention, car elle est le lieu de modifications intéressantes dans les éditions ultérieures : c'est le réseau dessiné par les mots soulignés d'un trait simple, articulé autour du sème /concavité $( \pm) /{ }^{(6)}$. Comme on le voit, celui-ci appartient également à une portion «non figurale » de la séquence, où la description se déroule selon une perspective plus topographique. A propos de cette isotopie, la connaissance des éditions ultérieures conduit à s'intéresser à un segment qui n'attire pas tout de suite l'attention en raison de son caractère plutôt conventionnel, c'est-à-dire la proposition « le vide était comblé ». En effet, dans ce cas, l'association syntaxique entre vide et comblé procède autant d'une attraction sémique que d'une collocation en langue. Si le mouvement de l'écriture a bien visé à déployer un réseau sémantique, il semble ici avoir également été comme contraint de se couler dans une forme tout à fait attendue ${ }^{(7)}$. Or de façon significative, cette forme subit une modification lors de la première réédition du texte :

A cause du brouillard, on ne voyait plus le village. Cet étage d'en bas où il était bâti, un linge de brouillard était jeté dessus, et le linge recouvrait tout, à part la pointe du clocher qui en sortait par une déchirure. Et plus loin, à l'endroit où s'ouvrait la vallée, le même voile s'étendait; ainsi le vide était caché. Mais on le devinait quand même. Et qu'il y avait l'air dessous, cela se devinait aussi, à cause d'un mouvement qui passait quelquefois, glissant à sa surface comme une vague sur le lac; puis un lambeau en était arraché, qui venait lentement vers nous. (Ramuz $1917: 47$ )

Plusieurs opérations de réécriture réagencent la description imagée, et au premier titre le fait que cette image, précisément, n'est plus introduite par la médiation du

(5) Là encore, c'est en fonction du cotexte que le sème/textile/ se manifeste ici dans les mots déchirure, s'étendre, s'attacher et arracher, en vertu de leur voisinage avec les noms linge, voile et lambeau ainsi que des relations syntactico-sémantiques que le texte établit entre eux. Qui plus est, l'emploi de ces mots évoque indéniablement des expressions associées à la notion de tissu (étendre le linge, attacher un ruban, etc.).

(6) Pour versant, le sème se manifeste par assimilation cotextuelle (si le mot était inclus dans le syntagme «versant d'une montagne », c'est au contraire le sème /convexité/ qui serait activé). 
marqueur comme ; la rupture sémantique se fait plus marquée et, en termes pragmatiques, cela a pour effet de conférer à l'image une sorte de statut « objectif», non explicitement lié à un point de vue, ce qui tend à l'imposer un peu plus. On verra que ce changement est probablement le germe de transformations ultérieures ; pour l'instant, arrêtons-nous sur les modifications survenues dans les troisième et quatrième phrases du segment, en 1914, fondues en une seule unité typographique en 1917. Cette réunion a assurément pour origine partielle la suppression de la seconde proposition indépendante dont la troisième phrase était constituée, ainsi que des composants (participiale, relative) gravitant autour de cette proposition ; on constate qu'un des résultats de cette modification est que le réseau /concavité $( \pm)$ / se voit réduit de moitié, et perd de son importance dans l'organisation sémantico-référentielle de cette partie de la séquence descriptive. Dès lors, la modification lexicale de comblé en caché paraît découler de cette suppression, montrant la relation que l'unité-mot entretient avec son cotexte immédiat, où prédomine alors l'isotopie /visibilité $( \pm)$ /. Indéniablement, au lieu de passer «naturellement » par l'association collocative entre vide et comblé, le mouvement de (ré)écriture a cette fois buté dessus, la faisant disparaître au profit d'une nouvelle forme, qui va « dans le sens » de ce que construit le paragraphe, à un niveau macrostructurel. La comparaison entre les deux états de texte montre par ailleurs que, en 1914, la phrase " Ainsi le vide était comblé » est complètement déconnectée des deux isotopies dominantes qui charpentent le paragraphe. Le travail de réécriture tend alors à réinscrire dans le tissu du texte une unité qui, sur ce plan-là, n'y tenait pas. Et l'on comprend alors que la fusion de deux phrases en une est également déterminée par la modification lexicale elle-même, car ces deux opérations conjointes aboutissent à renforcer la relation sémantique de causalité qui structure l'enchaînement des propositions (voile ainsi caché).

Nouvelle étape de la genèse de cette description, l'édition de 1922 offre une version du segment encore une fois réduite, et qui plus est avec une variation lexicale déterminante :

A cause du brouillard, on ne voyait plus le village. L'étage où il était bâti, un linge de brouillard avait été jeté dessus, et le linge recouvrait tout, à l'exception du clocher qui en sortait par une déchirure. Mais, par l'effet de l'air qu'il y avait dessous, parfois la toile se mettait à bouger, un mouvement passait à sa surface, comme une vague sur le lac, et un lambeau s'en détachait, qui venait lentement à vous. (Ramuz $1922: 40$ )

Là encore, le paragraphe est remanié dans son entier, et l'on constate que la description topographique et son réseau /concavité $( \pm)$ / ont totalement disparu. Mais ce que l'on note surtout, c'est le renforcement de l'isotopie articulée autour du trait /textile/, par l'apparition du nom toile, dont on a envie de dire qu'il remplace voile, quand bien même (à cause de la refonte du segment) le mot n'apparaît pas exactement à la même place que le précédent. Toutefois, il reste l'antécédent de l'anaphore possessive «sa surface» ${ }^{(8)}$, ce qui indique que les syntagmes « le même voile» et « la toile » partagent un statut textuel identique ; qui plus est, ces deux unités servent à référer à l'objet-brouillard au regard du même procès (la mise en mouvement). Et pardessus tout, comment ne pas penser que le second mot surgit sous la plume comme substitut du premier, dans la mesure où leurs signifiants respectifs se ressemblent au

(7) La base Frantext donne ainsi plus de 200 occurrences d'une association entre les deux mots... en ne comptant que les formes combler et comblé.

(8) Notons que cette modification a pour conséquence que le mot surface ne participe plus du tout du réseau/visibilité/. 
point qu'il a suffi de permuter un seul graphème afin de passer de l'un à l'autre ? La réécriture joue ici avec la deuxième articulation du langage, exploite le caractère distinctif des graphèmes pour altérer subtilement l'organisation sémantique du paragraphe, puisque le passage de / $/$ à /t/ dans cette suite de lettres a pour corollaire la disparition, en ce point du texte, du sème /visibilité/, au profit exclusif du sème/textile/, ce qui renforce la cohésion de la fin du segment de texte, par la redondance de ce trait-là. Comme je l'annonçais, cette modification réévalue la dimension polyisotopique du nom voile et son haut potentiel cohésif, très probablement en fonction d'une intention de renforcer l' "image » déployée dans ce paragraphe, de lui conférer le poids d'un donné perceptuel qui s'impose sans médiation, occultant une perception non filtrée par l'analogie. Or une telle intention paraît demander d'insister sur le décalage sémantique qui sert de moteur à la production d'une figure du type de la métaphore, ce que certains changements opéraient déjà en 1917. La substitution de toile à voile parachève alors ce mouvement de réécriture, puisque voile réalisait une fusion entre les réseaux non figural et figural, alors que toile, comme linge, consomme la rupture sémantique ${ }^{(9)}$.

\section{Le mot pivot : stratification de l'étiquette sémantique et composantes de cohésion du texte}

Lorsque la réécriture a pour résultat de resserrer le texte, il est somme toute assez naturel que ce mouvement aboutisse à une sélection parmi les isotopies plurielles, afin de condenser le sens d'un segment. Mais il va de soi que la réécriture peut aller dans la direction opposée, celle de l'expansion, de la diversification des réseaux; par ailleurs, comme le montre le cas emblématique du nom voile, la variation, plutôt que de viser à la « simplification » sémantique, peut au contraire faire émerger le mot qui, par sa structure sémantique propre, permet en un lieu donné le croisement de diverses composantes de la cohésion textuelle. Je me concentrerai sur deux cas de figure, parmi d'autres.

\subsection{Textualité et hétérogénéité énonciative : la cohésion stylistique}

Les traités de rhétorique accordent une grande attention à ce qui, dans le domaine de l'elocutio, détermine le choix des mots, qui doit être gouverné par le principe de convenance, c'est-à-dire par le souci d'une adéquation entre le style et les divers paramètres de la situation de discours. Parmi ceux-ci, l'objet du discours est un des premiers critères à considérer, comme le souligne Cicéron, selon qui, en matière de convenance, « la seule règle [...] à donner ici est de choisir, parmi les genres d'éloquence [...], celui qui convient le mieux au sujet» (Cicéron 1971:88). Mais, plus largement, cette convenance du style procède de normes génériques, puisque « les discours politiques, les panégyriques, les plaidoyers ne veulent pas le même style, non plus que les passages familiers ou soutenus, l'invective, l'exposé théorique, l'anecdote » (ibidem).

Dans la droite ligne des conceptions de la tradition rhétorique, les thèses développées par (ou attribuées à) Bakhtine, on le sait, ont grandement contribué à notre compréhension du rôle joué par les genres dans la structuration d'une parole singulière, en mettant au jour notamment l'ancrage de tout énoncé dans une sphère sociale d'échange (Bakhtine 1984 : 265). Ceci détermine l'inscription d'une parole dans un

(9) L'usage du mot voile ou de ses dérivés participe par ailleurs de collocations lorsqu'il s'agit de décrire des phénomènes atmosphériques (ciel voilé, voile de brume, etc.). 
genre, et par là contraint plusieurs paramètres de la constitution d'un énoncé, que ce soit son contenu thématique, sa construction compositionnelle ou son style (ibidem). Ce dernier point nous concerne particulièrement, car il touche notamment aux choix lexicaux qu'un locuteur est amené à faire et qui vont influer sur la progression de son discours :

Lorsque nous choisissons un mot, au cours du processus d'élaboration d'un énoncé, nous ne le prenons pas toujours, tant s'en faut, dans le système de la langue, dans sa neutralité lexicographique. Nous le prenons habituellement dans

d'autres énoncés, et, avant tout, dans des énoncés qui sont apparentés au nôtre par le genre, c'est-à-dire par le thème, la composition et le style : nous sélectionnons les mots selon les spécificités d'un genre. Le genre du discours n'est pas une forme de la langue, mais une forme de l'énoncé qui, en tant que tel, reçoit du genre une expression déterminée, typique, propre au genre donné. [...]

Il est possible d'assimiler cette expression typique du genre à l' "aura stylistique" du mot [...]. Ce qu'on entend résonner dans le mot, c'est l'écho du genre dans sa totalité. (Bakhtine 1984 : 294-295; je souligne en gras.)

Or si le processus de sélection des mots est à ce point sensible à leur « aura stylistique », à leur adéquation au genre choisi pour développer l'énoncé, c'est que l'apprentissage même de la langue ne se fait «pas dans les dictionnaires et les grammaires ", mais «à travers des énoncés concrets que nous entendons et que nous reproduisons au cours de l'échange verbal vivant qui se fait avec les individus qui nous entourent » (idem : 285). La mémoire lexicale est ainsi structurée par la diversité générique, ce qui a pour résultat de produire, au sein du stock de mots d'une langue, des oppositions sur la base de ces différences d'ancrages génériques et socio-discursifs, ce que manifeste l' " aura stylistique » qui nimbe le mot et l'empêche de se présenter à nous comme "neutre », non marqué par les spécificités d'une sphère d'échange.

Conçue de cette manière, la dimension stylistique participe immanquablement des forces cohésives qui président à l'organisation du texte. Le jugement de textualité - par quoi une suite de phrases est appréhendée comme une entité-texte - se fonde en effet également sur une évaluation de la bonne formation d'un discours en fonction de son rattachement à un genre ou à une famille de genres; autrement dit, on en revient à la question de la convenance du style. Certes, un « accroc » stylistique ne met pas en péril la cohésion du texte de la même manière qu'une gestion hasardeuse de la progression thématique ou un usage flottant des connecteurs... Il n'empêche que ce type de rupture dans le continu du texte nuit au sentiment d'avoir affaire à un objet doté de son unité. La raison en est que la cohésion stylistique est en réalité un avatar de la cohésion sémantique du texte, plus précisément de la part du sens qui procède, précisément, de la reconnaissance d'un ancrage discursif, et qui variera selon qu'un faisceau de traits indiquent que je lis un roman comique ou une tragédie, que c'est un ouvrier métallurgiste ou un ingénieur polytechnicien qui me parle, etc. De sorte que, au nombre des réseaux sémantiques qui structurent un texte, il faut bien tenir compte de ce que l'on appellera avec Kerbrat-Orecchioni des isotopies stylistiques (Kerbrat-Orecchioni 1977 : 97-98), ou de ce que Rastier désigne pour sa part comme des isotopies mésogénériques, construites par la récurrence d'un sème renvoyant à un "domaine » défini, classe structurant « la représentation linguistique d'une pratique sociale codifiée » (Rastier 1989 : 55) et correspondant à un « discours $»-$ juridique, médical, etc. (idem : 39-40).

La part du travail d'écriture qui vise à la cohésion stylistique se confronte dès lors à la nature fondamentalement dialogique de toute communication humaine, et à l'hétérogénéité énonciative qui en découle, sur fond de laquelle repose un texte; celui-ci 
est potentiellement traversé en chaque point de son déroulement par des discours aux origines diverses, en vertu notamment des unités lexicales qui pourraient ou non l'intégrer, et parmi lesquelles il s'agit d'opérer un tri. Pour ce qui touche aux textes à dominante narrative, ce "lissage » de l'hétérogène ou, au contraire, sa mise en évidence prend particulièrement place dans la distinction et l'articulation des différentes « voix » dont le récit se compose, celles du (des) narrateur(s) et des personnages. L'effet de texte (comme tout cohésif) se joue ici entre, d'une part, la construction cohérente d'une portion du monde fictionnel et, d'autre part, l'expression d'un ancrage énonciatif homogène par un biais stylistico-sémantique, de manière à manifester la perspective à travers laquelle cette portion de monde est représentée. Or, là aussi, l'observation de réécritures met au jour les tensions existant entre ces divers facteurs de liage, comme dans cet autre segment descriptif, dont voici le premier état publié :

[Lhôte] s'était redressé, et [Marguerite] voyait ses beaux grands yeux noirs briller à la lueur de la chandelle. Elle voyait que c'était son fils et qu'il était grand et beau. Elle voyait que ses habits étaient tout trempés de neige fondue et il y avait à ses genoux deux larges ronds d'humidité. Des gouttes pendaient dans sa barbe. (Ramuz 1914, $2:$ 786)

Si l'on se concentre sur la deuxième partie de la description, on constate que celleci repose en particulier sur l'isotopie/liquide/, voire/aqueux/, déployée à travers les mots trempés, neige, fondue, humidité et gouttes. Dans ce co(n)texte, le syntagme « ronds d'humidité » se présente même comme une forme tout à fait attendue, genoux appelant ronds, et humidité apparaissant comme le mot par excellence pour signifier un état d'imprégnation par un liquide. De même que pour la proposition « le vide était caché » abordée ci-dessus, mais pas pour les mêmes motifs ${ }^{(10)}$, le processus d'écriture semble avoir passé par une sorte de lieu quasi obligé. Pourtant la pertinence de cette forme va se voir précisément réévaluée à la baisse lors d'une deuxième phase de la genèse éditoriale :

[Lhôte] s'était redressé, et [Marguerite] voyait ses beaux grands yeux noirs briller à la lueur de la chandelle. [...] Elle voyait que ses habits étaient tout trempés de neige fondue et il avait à ses genoux deux larges ronds de mouillé. Des gouttes pendaient après sa barbe. (Ramuz 1917 : 125)

La variation lexicale ne met pas en jeu le sème /liquide/, car il compose au même titre les signifiés respectifs de mouillé et d'humidité. En revanche, elle est un geste fort, visant incontestablement la démarcation lexicale, puisqu'elle nous fait transiter d'une forme attendue à un néologisme, à travers l'emploi de mouillé non comme adjectif mais comme nom ${ }^{(11)}$. Or cette substitution doit être avant tout interprétée dans une perspective stylistique d'adéquation des mots aux choses ${ }^{(12)}$. Un faisceau de

(10) C'est bien le cotexte qui motive l'apparition d'un tel segment; il ne s'agit pas là d'une collocation (la base Frantext ne signale aucune occurrence du syntagme ; le Trésor de la langue française indique quant à lui comme formations synthétiques les associations « tache d'humidité » ou «trace d'humidité »).

(11) Si l'emploi est attesté par le Trésor de la langue française, on n'en trouve qu'une vingtaine d'occurrences dans Frantext ; plusieurs d'entre elles apparaissent dans des textes faisant la part belle à un parler populaire (Colette, Céline, Sand, Zola...). Signalons que les dictionnaires usuels réservent en revanche une entrée au nom mouillure.

(12) La variation touche bien entendu à d'autres composants sémantiques; humidité réfère ainsi à une propriété, alors que mouillé (dérivé d'un verbe) signale également que cette propriété est le résultat d'un procès, d'une transformation, ce qui est par ailleurs aussi le cas de trempés (il y a donc un fait de redondance entre les deux termes). L'analyse cependant ne peut pas s'en tenir à ce constat, à cause notamment de l'existence du nom mouillure. 
marques ${ }^{(13)}$ indiquent en effet que le segment descriptif a pour objectif (entre autres) de manifester le point de vue de la vieille Marguerite (rendue inquiète par l'absence de son fils). De ce fait, si nous n'avons pas affaire à une séquence de discours rapporté proprement dite, on peut toutefois estimer que le texte ici est (ré)élaboré de manière à produire un " discours approprié à l'objet du dire ", c'est-à-dire un discours que « le discours en train de se faire [...] rencontre comme "dans" l'objet qu'il vise» (Authier-Revuz 1995:316) - ici le personnage focal, qui participe d'un milieu populaire et campagnard. Si le remplacement d'humidité par mouillé sert une telle entreprise, c'est qu'il introduit un néologisme formé en usant du mécanisme de la conversion, grâce auquel Ramuz cherche de toute évidence à évoquer une parole paysanne ou populaire - et cela en pariant justement sur notre connaissance d'un mot équivalent comme humidité. Comme je l'ai dit, ce dernier est complètement attendu ; dès lors, le fait que cette attente ne soit pas remplie et que survienne au contraire un terme « inconnu » formé par conversion mime le déroulement du discours d'un locuteur qui forge un nom à partir d'une autre base lexicale à sa disposition en raison soit de lacunes de son vocabulaire, soit d'une « habitude » sociolectale. Un tel phénomène semble en effet caractériser la langue populaire, empiriquement ${ }^{(14)}$, ce qui est peut-être évoqué ici ; mais on peut aussi voir là un procédé de représentation d'une parole de paysans ou de gens du peuple qui, comme d'autres qui lui sont apparentés, se fonde sur une conception stéréotypée qui veut que de tels locuteurs soient dotés d'une compétence linguistique limitée, notamment en termes de lexique. Quoi qu'il en soit, et par contrecoup, la variation fait ressentir le nom humidité comme étant socialement accentué, puisque, alors qu'il est attendu, il paraît " échapper » au point de vue d'un paysan ; résonne alors dans le mot l' « écho » de la famille de genres techniquesscientifiques propres notamment au discours météorologique ou climatologique. On constate encore que Ramuz renforce l'effet de caractérisation stylistique au moyen d'une substitution parallèle de dans par après, convoquant ici un usage de la préposition pour exprimer une relation exclusivement spatiale, que le Trésor de la langue française qualifie de populaire. Ainsi, de façon subtile, de telles variations font apparaître dans ce segment la superposition et l'articulation de deux pans de cohésion sémantique, l'un relevant du domaine référentiel (construction du jeune homme décrit) et l'autre du domaine énonciatif (construction de la subjectivité à travers laquelle le monde est restitué).

\subsection{Architecture du récit : le balisage séquentiel}

La mise en variation du lexique en fonction d'un facteur de cohésion stylistique est, on le voit, une illustration particulière du caractère stratifié de la cohésion du texte. Ce ne sont pas tant les étendues respectives des diverses forces à l'œuvre (de la collocation au niveau propositionnel ou syntagmatique au réseau sous-tendant un segment entier de texte) qui sont en jeu dans ce cas qu'une exploitation du « feuilleté » de l'étiquette sémantique même des mots, où des éléments de sens se distinguent par leur fonction (construire une référence, construire un point de vue, un ancrage discursif). Par ailleurs, c'est encore leur aspect plus ou moins explicite/implicite qui les différencie ; ce qui relève de l' " aura stylistique » participe essentiellement d'un sens implicite, indirect, qui - à moins de faire l'objet d'une monstration par le biais

(13) En suivant Rabatel (1998: 41 sqq. et 62 sqq.), on pointera le fait qu'il s'agit d'un segment participant de l'arrière-plan du texte, essentiellement à l'imparfait, ainsi que la présence d'un sujet percevant associé à des verbes de perception.

(14) «Le français permet aux mots de changer de catégorie grammaticale, ce que la langue populaire pratique plus librement que la langue normée » (Gadet 1992 : 107). 
d'une modalisation autonymique ( $c f$. Authier-Revuz 1995) - demande un effort interprétatif plus important afin d'être saisi (ce qui explique bien entendu que certaines nuances stylistiques passent parfois inaperçues, ou sont sujettes à discussion). Or il existe d'autres composants du signifié des unités lexicales qui, eux aussi, contribuent à stratifier ce dernier et procèdent de l'implicite linguistique : les présupposés. Si l'on se rappelle la description que donne Ducrot des deux grandes formes (selon lui) d'implicite, c'est-à-dire les présupposés et les sous-entendus, les premiers se caractérisent par le fait qu'ils font "partie de la signification littérale des énoncés » (Ducrot 1998 : 24). Ainsi, à la différence des sous-entendus, qui ne sont rétablis qu'à partir d'un certain contexte de communication, les présupposés, inscrits dans la structure d'un énoncé, disposent de supports, et en particulier de supports lexicaux.

Dans le cadre d'un récit, le mécanisme de l'implicite intervient bien entendu à différents niveaux, et permet en particulier d'introduire des ellipses, que ce soit à des fins d'économie de la narration ou de jeu rhétorique. Mais, pour ce qui est notamment des présupposés inclus dans les supports lexicaux, ceux-ci participent également aux liages textuels dans la mesure où ils réalisent une forme de redondance, par rappel d'éléments déjà posés dans le cotexte en amont. Observée sous cet angle, l'unité lexicale se présente alors comme un pivot textuel particulièrement intéressant, puisqu'elle articule en son sein (potentiellement) les deux principes de base de la textualité que sont les exigences contraires de progression et de répétition ( $c f$. notamment pour le récit Adam 1985: 42 sqq.).

Comme la dynamique narrative procède en bonne part de la représentation d'événements sous la forme d'un enchaînement temporel et causal, il est indéniable que l'élaboration d'un récit demande d'accorder une certaine attention au réglage de cet enchaînement. Se pose ainsi la question des séquences narratives et de leurs étapes propres, ainsi que de la transition de l'une à l'autre, nécessitant un marquage tant de la séquence et de son étendue que des macropropositions narratives qui la constituent, afin que le texte et son déroulement apparaissent comme planifiés par la chrono-logie de l'action ( $c f$. Adam 1985). On associe de façon préférentielle les organisateurs temporels ou les connecteurs argumentatifs à cette fonction de balisage; or il se trouve qu'un certain nombre de verbes, eux aussi, endossent idéalement un tel rôle : ce sont les verbes aspectuels ou transformatifs, qui par leur nature même signalent le commencement, la poursuite ou la fin d'un procès. En voici un exemple, dans un passage où des villageois (qui ont accepté le diable comme maître) veulent s'emparer d'une jeune fille (dont la pureté va être fatale au Malin) :

Cependant tous les autres se levaient, et tous ces autres s'avançaient à leur tour, ils disaient : « Laissez-nous-en! » alors Labre et Gentizon comprirent qu'ils n'avaient plus de temps à perdre. Gentizon se décida, Gentizon prit son élan. Gentizon roula par terre.

Il devait avoir mal visé : rien d'étonnant, pensait-on, dans l'état où il était; Labre le suivait de tout près, on se dit : «C'est lui qui l'aura. » Et il semblait avoir mieux calculé son geste : alors on ne sut plus ce qui arrivait ; mais voilà qu'à l'instant qu'il allait la rejoindre, ce fut comme si Labre se heurtait à un mur, Labre tomba à la renverse.

Alors les autres s'arrêtèrent : tout fit silence sur la place. Et c'est à la faveur de ce silence, que la rumeur, qui venait du village, se fit entendre de nouveau. (Ramuz $1922: 175-176)$

Avec le verbe $s$ 'arrêter, nous avons affaire à un véritable prototype de présupposition prenant appui sur un support lexical, car la notion véhiculée par le mot implique un procès préalablement en cours, qui dans le cas présent est donc posé explicitement 
dans le cotexte en amont («tous ces autres s'avançaient»). En sollicitant de cette manière notre mémoire discursive, en nous invitant à « remonter» dans le texte pour saisir toute l'étendue du sens convoqué à ce moment par le verbe s'arrêter, celui-ci (et son présupposé) est bien un facteur de liage textuel, opérant à la manière d'une anaphore, par exemple - tout comme le font par ailleurs les verbes se lever et s'avancer, au début de l'extrait, dont les présupposés respectifs /état antérieur allongé/ et /statisme préalable/ convoquent des éléments du cotexte amont concernant les personnages mis en scène. Il faut encore remarquer que la force cohésive du verbe interagit ici avec deux autres composantes : d'une part une marque de planification sous la forme de l'organisateur alors, qui signale lui aussi une étape séquentielle et associe une opération de segmentation (nouvelle étape) et une opération de liage (l'étape - et le texte - suit un ordre); d'autre part la forte segmentation introduite par le passage à un nouveau paragraphe, ponctuation de texte dont on sait qu'elle oriente fortement la saisie du sens, dans la mesure où «l'alinéa signale au lecteur qu'il vient de traiter une unité de sens et qu'il va passer à une unité ultérieure » (Bessonnat 1988: 85).

Nous sommes donc ici dans un lieu particulièrement sensible où doit se négocier la tension entre progression et répétition ; la version de 1922 propose une solution, qui se voit modifiée en 1937, dans un effort particulièrement frappant de ménager la transition entre le déjà-dit et le thème que le nouveau paragraphe a pour tâche de développer :

Cependant, tous les autres se levaient, et tous ces autres s'avançaient à leur tour, disant : «Laissez-nous-en ! » [...] Gentizon se décida, Gentizon prit son élan. Gentizon roula par terre.

Il devait avoir mal visé : rien d'étonnant, pensait-on, dans l'état où il était ; Labre le suivait de tout près $[\ldots]$. Labre tomba à la renverse.

Les autres s'étaient tus; tout fit silence sur la place. Et c'est à la faveur de ce $s i$ lence, que la rumeur, qui venait du village, se fit entendre de nouveau. (Ramuz $1937: 35-36)$

A l'égal du verbe s'arrêter, la forme se taire implique un procès préalable en cours, dont on voit que, là encore, le texte porte la trace en amont (à quoi s'ajoute probablement l'image d'un brouhaha de voix diverses, non représenté explicitement, mais que le verbe évoque par le même biais de la présupposition). Le liage par rappel d'un élément antérieur opère ainsi avec se taire ; on voit cependant aisément de quelle manière le verbe s'oriente simultanément vers le cotexte en aval, dont il renforce la cohésion sémantique, puisqu'il devient la première maille du réseau (indiqué par l'italique) formé autour du sème /sonorité ( \pm )/ qui sous-tend tout le paragraphe ${ }^{(15)}$. De sorte que, dans ce cas, la variation met au jour le jeu existant entre deux facteurs de liages textuels, que le mot peut avoir à charge - ou non - d'articuler : la cohésion procédant d'une isotopie active au sein d'une unité textuelle d'un certain rang (le paragraphe), et celle qui relève du marquage d'un ordre dérivant tant d'une « logique » de l'action que d'une structuration du texte, et renvoyant dès lors à une unité de rang supérieur (la séquence).

L'observation de tels exemples nous donne plusieurs indications sur la place oc-

(15) Le changement de tiroir verbo-temporel n'est également pas anodin : le passé simple en 1922 fait du procès dénoté par s'arrêter un élément de premier plan, alors que le plus-queparfait en 1937 relègue le procès dénoté par se taire à l'arrière-plan ; 1'événement étant représenté depuis la perspective d'un moment M équivalant à celui du procès dénoté dans la proposition suivante, «tout fit silence», ce dernier devient de ce fait un «centre de gravité » événementiel. 
cupée par le(s) mot(s) dans le déploiement du texte... ou plutôt dans son incessant redéploiement. L'approche génétique ne cesse en effet d'établir à quel point « une œuvre est une esquisse, un potentiel qui s'actualise à travers ses réalisations particulières, [...] toujours en train d'advenir ou d'exploiter, en bougeant, ses propres virtualités » (Jeanneret $1994: 26)$. Formulée d'abord pour décrire les pratiques littéraires au Moyen Age, cette remarque vaut néanmoins pour n'importe quelle production textuelle, tout genre et toute époque confondus. Or ce que montre encore l'étude des variations lexicales, c'est que ces virtualités en attente d'être concrétisées ont (en partie) pour origine la tension qui existe entre les diverses forces assurant la cohésion textuelle, et qui pèse notamment sur certains mots. Elaboré à travers des opérations contraires de segmentation (en propositions, en paragraphes...) et de liage, soumis aux exigences opposées de répétition et de progression, destiné à construire un tout homogène à partir de l'hétérogène (pluralité des réseaux sémantiques, hétérogénéité énonciative), le texte est nécessairement une structure en équilibre instable, dont les pans tantôt se soudent, tantôt se chevauchent, tantôt laissent des ouvertures à combler ou - pourquoi pas? - à élargir. De sorte que le mouvement de l'écriture le conduira invariablement... à varier.

\section{Références}

ADAM, J.-M. (1985) : Le Texte narratif. Traité d'analyse textuelle des récits, Paris, Nathan.

- (1999) : Linguistique textuelle. Des genres de discours aux textes, Paris, Nathan.

Authier-Revuz, J. (1995) : Ces mots qui ne vont pas de soi, 2 vol., Paris, Larousse. BAKHTINe, M. (1984 [1952-1953]) : Esthétique de la création verbale, Paris, Gallimard.

BESSONNAT, D. (1988) : «Le découpage en paragraphes et ses fonctions », Pratiques, 57, pp. 81-105.

CiCÉRON (1971 [1930]) : De l'orateur, livre III, texte établi par H. Bornecque et traduit par E. Courbaud et H. Bornecque, Paris, Belles-Lettres.

DoqueT-LACOSTE, C. (2008) : «Approche contextuelle du sens et activités lexicologiques à l'école élémentaire », in Grossmann, F. \& Plane, S., Les Apprentissages lexicaux. Lexique et production verbale, pp. 125-140.

Ducrot, O. (1998 [1972]) :Dire et ne pas dire. Principes de sémantique linguistique, Paris, Hermann.

FRANTEXT, base textuelle informatisée, http ://www.frantext.fr.

GADET, F. (1992) : Le Français populaire, Paris, PUF.

GRÉSILLON, A. (1988) : «Les manuscrits littéraires : le texte dans tous ses états », Pratiques, 57, pp. 107-122.

JEANNERET, M. (1994) : «Chantiers de la Renaissance. Les variations de l'imprimé au $\mathrm{XVI}^{\mathrm{e}}$ siècle », Genesis, 6, pp. 25-45. 
Kerbrat-OrecCHIOni, C. (1977) : La Connotation, Lyon : Presses universitaires de Lyon.

RABATEL, A. (1998) : La Construction textuelle du point de vue, Lausanne/Paris, Delachaux et Niestlé.

RAMuZ, C. F. (1914 (1-4)) : Le Règne de l'esprit malin, Mercure de France, 407 à 410, pp. 533-572, pp. 753-795, pp. 88-124, pp. 333-351.

- (1917) : Le Règne de l'esprit malin, Lausanne, Cahiers vaudois.

- (1922) : Le Règne de l'esprit malin, Genève, Georg.

- (1937) : Le Règne de l'esprit malin, Le Chef-d'Euvre. Organe mensuel de littérature, 5, pp. 5-37.

- (2005) : Journal, tome 3 (1921-1947), Genève, Slatkine.

RASTIER, F. (1987) : Sémantique interprétative, Paris, PUF.

— (1989) : Sens et textualité, Paris, Hachette.

TRÉSOR DE LA LANGUE FRANÇAISE, version informatisée,

http://www.atilf.atilf.fr/tlf.htm.

\section{Annexe : segments du Règne de l'esprit malin analysés}

\section{Segment 1 (chapitre II, version de 1914, de 1917 et de 1922)}

[termes en gras : isotopie constituée autour du trait /visibilité $( \pm) /$; termes en italique, isotopie /textile/; termes soulignés d'un trait simple : isotopie /concavité $( \pm) /]$

1a. A cause du brouillard, on ne voyait plus le village. Cet étage d'en bas, où il était bâti, était recouvert comme d'un linge, sous lequel tout disparaissait, à part la pointe du clocher, qui en sortait par une déchirure. Et plus loin, à l'endroit où s'ouvrait la vallée, le même voile s'étendait, et, partant de ce versant-ci, allait s'attacher au versant d'en face, dont il ne laissait voir que la partie d'en haut. Ainsi le vide était comblé. Mais on le devinait quand même. Et qu'il y avait l'air dessous, cela se devinait aussi, à cause d'un mouvement qui passait quelquefois, glissant à sa surface comme une vague sur un lac ; puis un lambeau en était arraché, qui venait lentement vers vous.

1b. A cause du brouillard, on ne voyait plus le village. Cet étage d'en bas où il était bâti, un linge de brouillard était jeté dessus, et le linge recouvrait tout, à part la pointe du clocher qui en sortait par une déchirure. Et plus loin, à l'endroit où s'ouvrait la vallée, le même voile s'étendait; ainsi le vide était caché. Mais on le devinait quand même. Et qu'il y avait l'air dessous, cela se devinait aussi, à cause d'un mouvement qui passait quelquefois, glissant à sa surface comme une vague sur le lac; puis un lambeau en était arraché, qui venait lentement vers nous.

1c. A cause du brouillard, on ne voyait plus le village. L'étage où il était bâti, un 
linge de brouillard avait été jeté dessus, et le linge recouvrait tout, à l'exception du clocher qui en sortait par une déchirure. Mais, par l'effet de l'air qu'il y avait dessous, parfois la toile se mettait à bouger, un mouvement passait à sa surface, comme une vague sur le lac, et un lambeau s'en détachait, qui venait lentement à vous.

\section{Segment 2 (chapitre IV, version de 1914 et de 1917)}

2a. [Lhôte] s'était redressé, et [Marguerite] voyait ses beaux grands yeux noirs briller à la lueur de la chandelle. Elle voyait que c'était son fils et qu'il était grand et beau. Elle voyait que ses habits étaient tout trempés de neige fondue et il y avait à ses genoux deux larges ronds d'humidité. Des gouttes pendaient dans sa barbe.

2b. [Lhôte] s'était redressé, et [Marguerite] voyait ses beaux grands yeux noirs briller à la lueur de la chandelle. [...] Elle voyait que ses habits étaient tout trempés de neige fondue et il avait à ses genoux deux larges ronds de mouillé. Des gouttes pendaient après sa barbe.

\section{Segment 3 (chapitre VII, version de 1922 et de 1937)}

[termes en gras : données rappelées par le présupposé compris dans le dernier verbe de la série mise en évidence ; termes en italique : isotopie constituée autour du trait /sonorité $( \pm) /$; terme souligné d'un trait simple : marqueur séquentiel]

3a. Cependant tous les autres se levaient, et tous ces autress'avançaient à leur tour, ils disaient : «Laissez-nous-en ! » alors Labre et Gentizon comprirent qu'ils n'avaient plus de temps à perdre. Gentizon se décida, Gentizon prit son élan. Gentizon roula par terre.

Il devait avoir mal visé : rien d'étonnant, pensait-on, dans l'état où il était ; Labre le suivait de tout près, on se dit: «C'est lui qui l'aura. » Et il semblait avoir mieux calculé son geste : alors on ne sut plus ce qui arrivait ; mais voilà qu'à l'instant qu'il allait la rejoindre, ce fut comme si Labre se heurtait à un mur, Labre tomba à la renverse.

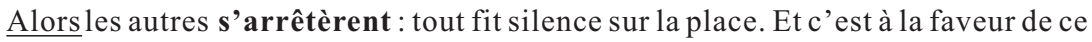
silence, que la rumeur, qui venait du village, se fit entendre de nouveau.

3b. Cependant, tous les autres se levaient, et tous ces autres s'avançaient à leur tour, disant : «Laissez-nous-en ! » [...] Gentizon se décida, Gentizon prit son élan. Gentizon roula par terre.

Il devait avoir mal visé : rien d'étonnant, pensait-on, dans l'état où il était; Labre le suivait de tout près $[\ldots]$. Labre tomba à la renverse.

Les autres s'étaient tus; tout fit silence sur la place. Et c'est à la faveur de ce $s i$ lence, que la rumeur, qui venait du village, se fit entendre de nouveau. 